\title{
Printed-Circuit Leaky-Wave Antenna With Pointing and Illumination Flexibility
}

\author{
José Luis Gómez-Tornero, David Cañete-Rebenaque, Student Member, IEEE, and \\ Alejandro Álvarez-Melcón, Member, IEEE
}

\begin{abstract}
A novel leaky-wave antenna, based on a periodic set of slots printed on a rectangular dielectric waveguide is conceived in this work. The antenna presents the ability to independently control the aperture illumination and the pointing direction, by only modifying the photoetched printed-circuit layout. The working mechanism is described using a leaky-mode dispersion analysis, and cosine-tapered designs are performed at $50 \mathrm{GHz}$. The radiation patterns are obtained using HFSS analysis to check the reduction of sidelobes levels, and the capacity to tune the pointing direction over a wide range of elevation angles.
\end{abstract}

Index Terms-Leaky-wave antennas, millimeter-wave antennas.

\section{INTRODUCTION}

$\mathbf{R}$ ECENTLY, a hybrid printed-circuit dielectric-waveguide technology has been proposed to conceive tapered leakywave antennas (LWA) for millimeter waveband applications [1]. In this technology, the originally nonradiative host dielectric guide becomes a leaky structure when a printed circuit is asymmetrically added in the dielectric-air interface. This technology also allows to taper the aperture illumination by modulating the planar circuit dimensions, leading to a reduction of the sidelobes level. A scheme of a slot-circuit tapered LWA is shown in Fig. 1(a). This type of antennas is mechanically easy to realize, since the slot dimensions are the only responsible for the antenna illumination. By interchanging different layouts over the same host guide, one can design a specified low-sidelobes radiation pattern, as demonstrated in [1]. However, the pointing direction of the LWA is mainly controlled by the dispersive frequency response of the $\mathrm{TE}_{10}$ mode of the host dielectric guide [1]. The only way to control the pointing angle of the LWA at a fixed frequency is by modifying the host guide dimensions [basically, the dielectric slab width $a$, see Fig. 1(a)]. In this work, a modification of this antenna is proposed. The new antenna makes use of a periodic set of slots printed on the dielectric guide [see Fig. 1(b)]. By adjusting the periodicity $P$ of the printed layout, the pointing direction of the antenna can be adjusted, without losing the ability to taper the aperture illumination.

\section{This}

work was supported by Spanish National Projects ESP2001-4546-PE, TEC2004-04313-C0202-TCM, Regional Seneca Project 2002 PB/4/FS/02, and the EPSON-Ibérica Foundation. The review of this letter was arranged by Associate Editor J.-G. Ma.

The authors are with Departamento de Tecnologías de la Información y las Comunicaciones, Technical University of Cartagena, Antiguo Hospital de Marina, Cartagena 30202, Spain (e-mail: josel.gomez@upct.es).

Digital Object Identifier 10.1109/LMWC.2005.852801

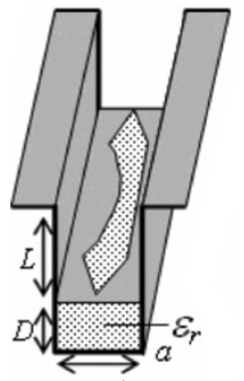

a)

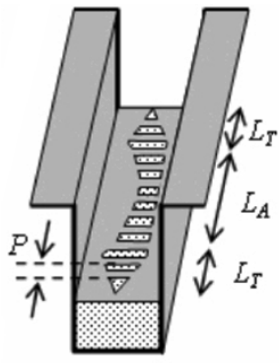

b)

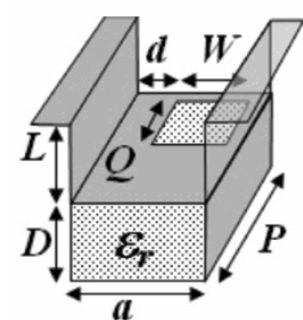

c)
Fig. 1. Hybrid printed-circuit dielectric-waveguide leaky-wave antennas (a) uniform, (b) periodic, and (c) unit cell.

\section{THEORY AND RESUlTS}

When the printed slot is modified from the uniform [Fig. 1(a)] to the periodic layout [Fig. 1(b)], the set of space-harmonics are excited in the periodic structure [2]. The propagation constant of the $m=-1$ space-harmonic can be described in complex form if this harmonic is radiating, as it corresponds to a leaky-wave

$$
k_{-1}=\beta_{-1}-j \alpha=\left\{\beta_{0}-\frac{2 \pi}{P}\right\}-j \alpha \quad\left(m^{-1}\right)
$$

where $\beta_{0}$ is the bloch-wave phase constant and $\alpha$ is the leakage rate. For the antennas shown in Fig. 1, $\beta_{0}$ is mainly determined by the waveguide width $a$, since the bloch-wave is the perturbation of the $\mathrm{TE}_{10}$ mode of the host dielectric waveguide. Conversely, the phase constant of the $m=-1$ space-harmonic, $\beta_{-1}$, can be modified by changing the printed-circuit periodicity $P\left(\beta_{-1}=\beta_{0}-2 \pi / P\right)$. The $m=-1$ harmonic radiates if its phase constant satisfies the radiation condition [2], given by

$$
\left|\frac{\beta_{-1}}{k_{0}}\right|<1
$$

where $k_{0}$ stands for the free-space wavenumber. In this case, the angle of maximum radiation $\theta_{m}$ is given by the next equation

$$
\theta_{m}=\arcsin \frac{\beta_{-1}}{k_{0}} \text {. }
$$

The elevation pointing angle $\theta_{m}$ is measured from the broadside direction, and it can have either positive or negative values, depending on the frequency of operation. As the frequency is increased, $\theta_{m}$ moves from the backward to the forward quadrant, leading to the well-known backward to forward frequency 


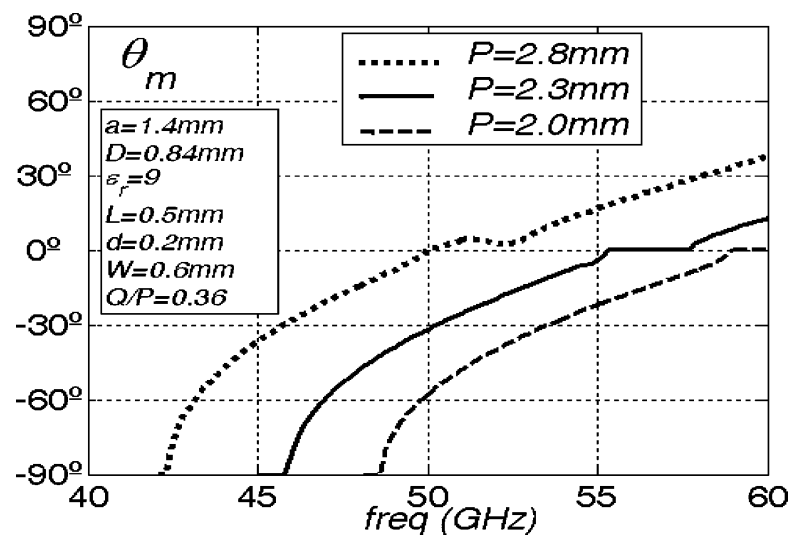

Fig. 2. Slitted PLWA millimeter waveband frequency-scanning response for different periodicities.

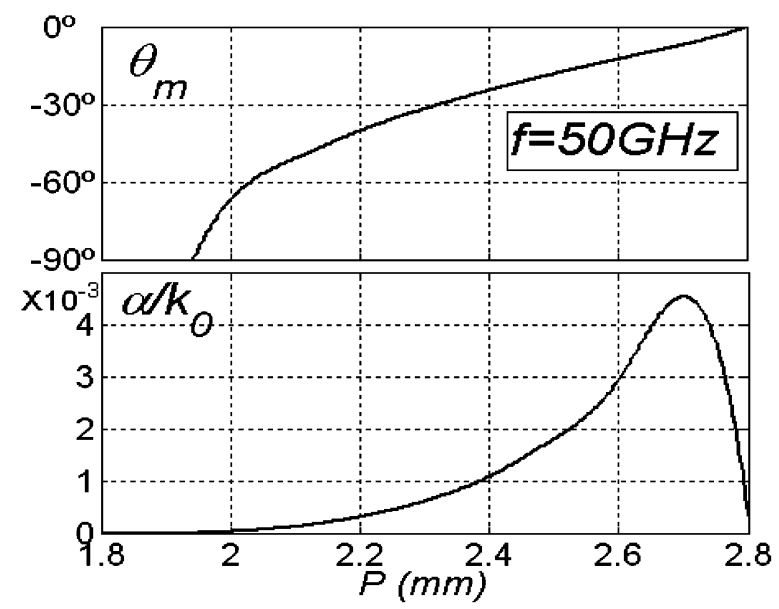

Fig. 3. Dependence of $\theta_{m}$ and $\alpha / k_{0}$ at $50 \mathrm{GHz}$ with $P$ for the slitted PLWA $(Q / P=0.36)$.

scanning response inherent to any periodic leaky-wave antenna (PLWA) [2].

A moment method accurate leaky-mode analysis method [3], specifically conceived for the type of structures shown in Fig. 1, has been applied to obtain the complex leaky-wave propagation constants. By applying periodic boundary conditions, the unit cell shown in Fig. 1(c) is analyzed to obtain the dependence of $\theta_{m}$ and $\alpha$ with the frequency, and with the different geometrical parameters.

Fig. 2 shows the frequency response of $\theta_{m}$ for the proposed slitted PLWA shown in Fig. 1(b), and for different values of $P$. The values of the rest of geometrical parameters, according to Fig. 1(c), are summarized in the inset of Fig. 2. It can be seen how the response is shifted to lower frequencies as the periodicity is increased. This phenomenon explains how, at a fixed frequency, the pointing angle can be increased by using a larger value of $P$. As an example, Fig. 3 shows the variation of $\theta_{m}$ at $50 \mathrm{GHz}$ for the same dimensions of the PLWA in Fig. 2, as a function of $P$. In this analysis we use a constant normalized slot length $(Q / P=0.36)$, and a linear dependence is obtained from $\theta_{m}=-60^{\circ}$ to $\theta_{m}=0^{\circ}$. The backward quadrant has been chosen to avoid the interference of higher-order radiating space-harmonic, which commonly occurs in the forward quadrant [2]. Also the variation of the normalized leakage

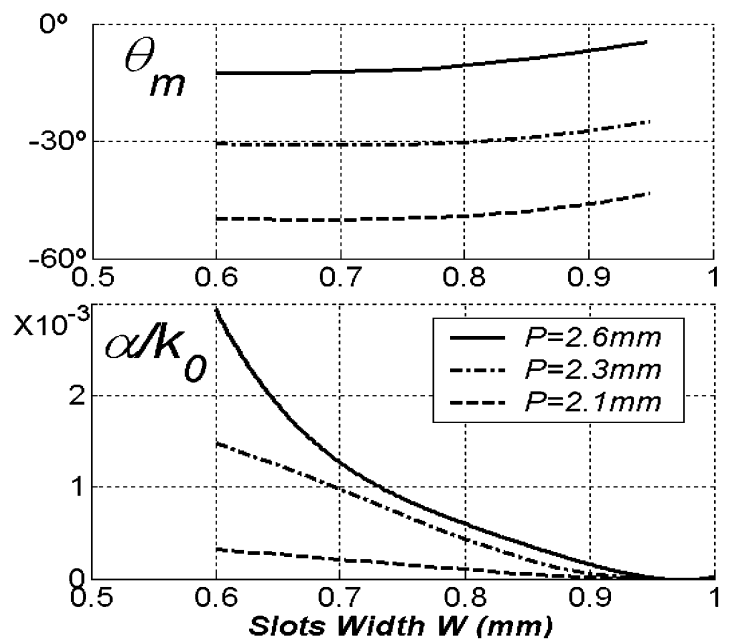

Fig. 4. Dependence of $\theta_{m}$ and $\alpha / k_{0}$ at $50 \mathrm{GHz}$ with the slot width for different values of $P(Q / P=0.36)$, with $d=0.2 \mathrm{~mm}$.

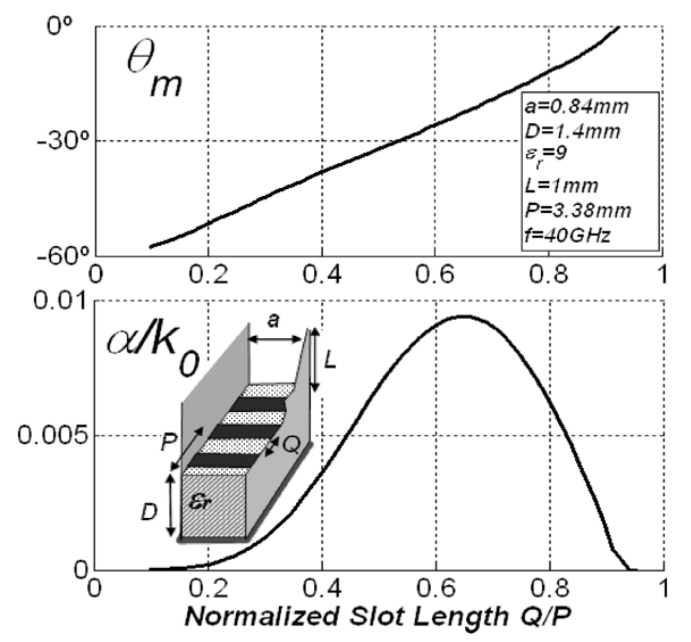

Fig. 5. Control of the leakage rate in conventional PLWA.

rate, $\alpha / k_{0}$, is plotted. As it is well-known, $\alpha / k_{0}$ decreases as the leaky-mode reaches the endfire direction $\left(\theta_{m}=-90^{\circ}\right)$.

Different technologies have been applied to conceive PLWA which present the same pointing-control behavior, as the microstrip [4], the dielectric grating [5], the strip-loaded dielectric slab [6] and the dielectric-inset waveguide [7]. However, the main contribution of the proposed antenna is that the leakage rate can also be controlled by using the same asymmetry radiation principle demonstrated in [1] for nonperiodic LWA. This control is performed by adjusting the asymmetry level, and it can be done for any value of $P$. Fig. 4 shows how $\alpha / k_{0}$ is varied from zero to a maximum value, when the slots width $W$ is modified. Analogous responses can be obtained by modifying the slot position $d$. Three values of $P$ have been selected, which result in three different pointing directions at $50 \mathrm{GHz}$ according to Fig. 3. In this way, $\alpha / k_{0}$ can be controlled without affecting the pointing direction $\theta_{m}$.

This leakage control mechanism is the main novelty with respect to other PLWA [6], [7] which were based on slots printed along the whole width of the host dielectric waveguide $(W=$ a). An example is given in Fig. 5, where the control of $\alpha / k_{0}$ for the dielectric-inset PLWA proposed in [7] is illustrated. 

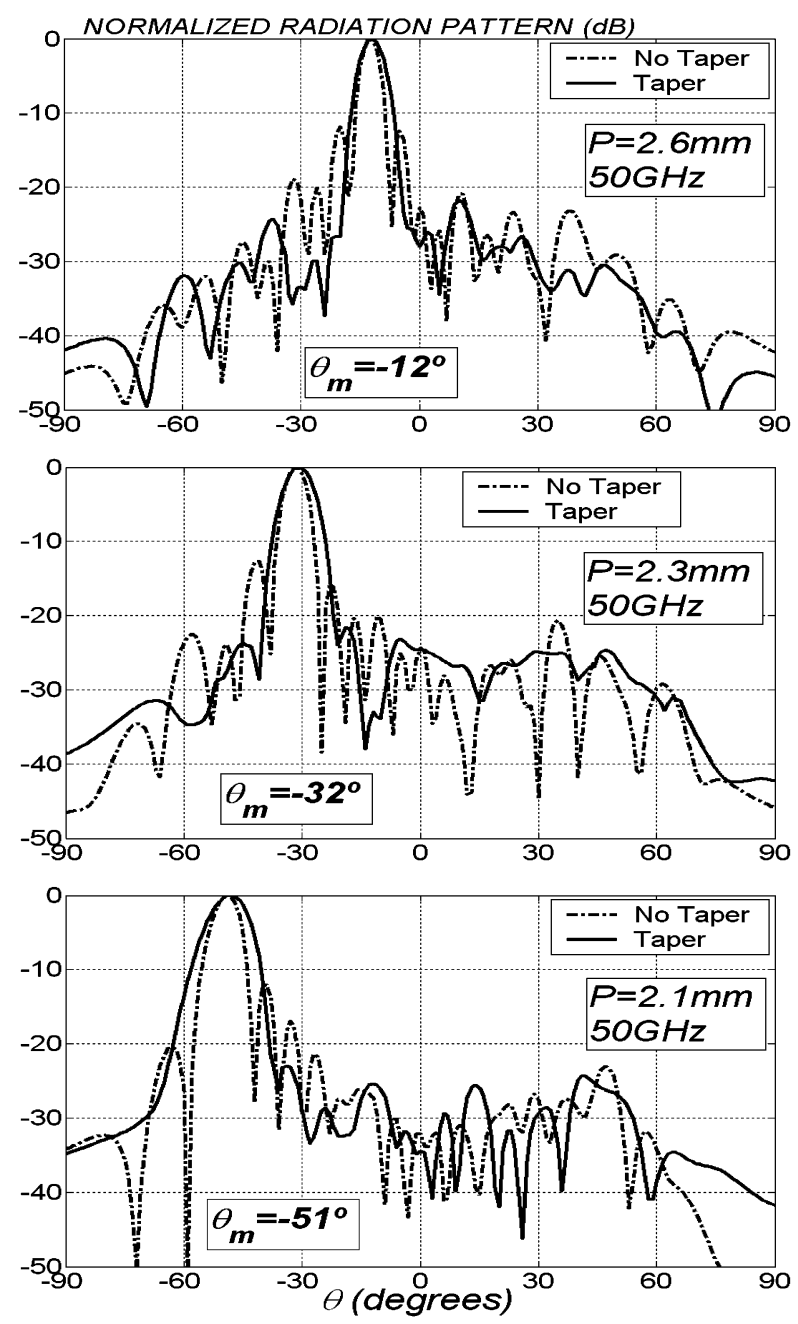

Fig. 6. Normalized radiation patterns obtained with HFSS, demonstrating the ability to control $\theta_{m}$ and the sidelobes level.

In all previously proposed tapered printed-circuit PLWA, $\alpha / k_{0}$ could only be controlled by modifying the slot length $Q$. As it can be seen in Fig. 5, this process has a strong effect in the pointing direction of the PLWA. In order to keep $\theta_{m}$ constant for all the values of $\alpha / k_{0}$, the period $P$ must also be varied for the different values of $Q$. This tapering procedure is much more complicated than in the case of the new printed-circuit PLWA, where $P$ need not be modified to make the taper.

To validate the mentioned ability to independently and easily control the pointing direction and the aperture illumination, three different designs have been performed. All of them work at $50 \mathrm{GHz}$ and use the same host dielectric waveguide than in previous figures $(a=1.4 \mathrm{~mm}, D=0.84 \mathrm{~mm}, \varepsilon r=9$, $L=5 \mathrm{~mm}$ ). The length of the antennas is $L_{A}=10 \lambda_{0}$. The periodicity $P$ has been selected to radiate at three different $\theta_{m}$, which cover a wide range of pointing directions. As it was illustrated in the leaky-mode dispersion curves of Fig. 4, the slots widths can be modified along the antenna length to vary the leakage rate $\alpha / k_{0}$ while barely affecting $\theta_{m}$. The small phase aberrations in $\theta_{m}$ which can be seen in Fig. 4 are corrected by readjusting the slots position [parameter $d$ in Fig. 1(c)] [1]. In this way, one can obtain the variation of the layout dimensions ( $W$ and $d$ ) along the antenna length to obtain a tapered cosine illumination in the antenna aperture for any pointing direction (selected with $P$ ).

The layouts designed with the leaky-mode analysis approach were introduced in a commercial electromagnetic analysis tool (HFSS). The results obtained for the normalized radiation patterns are plotted in Fig. 6. The control of the pointing direction is confirmed, obtaining the values predicted by the leaky-mode analysis $\left(\theta_{m}=-12^{\circ},-32^{\circ}\right.$ and $-51^{\circ}$ for $P=2.6 \mathrm{~mm}$, $2.3 \mathrm{~mm}$, and $2.1 \mathrm{~mm}$, according to Fig. 3). Both the tapered and the nontapered antennas were analyzed to check the reduction of the sidelobes level due to the cosine illumination. It can be seen in Fig. 6 that the sidelobes are reduced from $13 \mathrm{~dB}$ to below $20 \mathrm{~dB}$ in all cases. Tapered transitions of length $L_{T}=2 \lambda_{0}$ [see Fig. 1(b)] were added at the edges of the printed-circuit to reduce reflections.

\section{CONCLUSION}

The proposed antenna allows to control both $\theta_{m}$ and $\alpha / k_{0}$ by only modifying the periodic slot layout, while the host dielectric waveguide dimensions do not need to be altered. This antenna presents important advantages with respect to the nonperiodic counterpart (in which $\theta_{m}$ could only be controlled by varying the frequency or the host guide geometry), and also as compared to previous printed periodic antennas (in which $\alpha$ could not be controlled without affecting $\theta_{m}$ ). This design flexibility has been checked with HFSS results. This technology also offers interesting manufacturing advantages, since different slots masks can be designed to obtain different desired electrical specifications (pointing direction and illumination), and lately incorporated to the same host waveguide.

\section{REFERENCES}

[1] J. L. Gómez, A. de la Torre, D. Cañete, M. Gugliemi, and A. A. Melcón, "Design of tapered leaky-wave antennas in hybrid waveguide-planar technology for millimeter waveband applications," IEEE Trans. Antennas Propagat., vol. 53, no. 8, Aug. 2005.

[2] A. A. Oliner, "Leaky-wave antennas," in Antenna Engineering Handbook, 3rd ed. New York: McGraw-Hill, 1993, ch. 10.

[3] J. L. Gómez and A. A. Melcón, "Radiation analysis in the space domain of laterally-shielded planar transmission lines. Part I: theory," Radio Science, vol. 39, no. 3, pp. 1-11, Jun. 2004.

[4] M. Ghomi and H. Baudrand, "Full-wave analysis of microstrip leakywave antenna," Electron. Lett., vol. 25, no. 13, pp. 870-871, 1989.

[5] F. Schwering and S. T. Peng, "Design of dielectric grating antennas for millimeter-wave applications," IEEE Trans. Microwave Theory Tech., vol. 31, no. 2, pp. 199-209, Feb. 1983.

[6] M. Mittra and R. Kastner, "A spectral domain approach for computing the radiation characteristics of a leaky-wave antenna for millimeter waves," IEEE Trans. Antennas Propagat., vol. 29, pp. 652-654, 1981.

[7] M. Guglielmi and G. Boccalone, "A novel theory for dielectric-inset waveguide leaky-wave antennas," IEEE Trans. Antennas Propagat., vol. 39, no. 4, pp. 497-504, Apr. 1991. 